\title{
Tuberculosis screening costs and cost-effectiveness in high-risk groups: a systematic review
}

\author{
H. Alsdurf ${ }^{1}$, B. Empringham ${ }^{1,2}$, C. Miller $^{3}$ and A. Zwerling ${ }^{1 *}$
}

\begin{abstract}
Background: Systematic screening for active tuberculosis (TB) is a strategy which requires the health system to seek out individuals, rather than waiting for individuals to self-present with symptoms (i.e., passive case finding). Our review aimed to summarize the current economic evidence and understand the costs and cost-effectiveness of systematic screening approaches among high-risk groups and settings.

Methods: We conducted a systematic review on economic evaluations of screening for TB disease targeting persons with clinical and/or structural risk factors, such as persons living with HIV (PLHIV) or persons experiencing homelessness. We searched three databases for studies published between January 1, 2010 and February 1, 2020. Studies were included if they reported cost and a key outcome measure. Owing to considerable heterogeneity in settings and type of screening strategy, we synthesized data descriptively.
\end{abstract}

Results: A total of 27 articles were included in our review; 19/27 (70\%) took place in high TB burden countries. Seventeen studies took place among persons with clinical risk factors, including 14 among PLHIV, while 13 studies were among persons with structural risk factors. Nine studies reported incremental cost-effectiveness ratios (ICERs) ranging from US\$51 to $\$ 1980$ per disability-adjusted life year (DALY) averted. Screening was most cost-effective among PLHIV. Among persons with clinical and structural risk factors there was limited evidence, but screening was generally not shown to be cost-effective.

Conclusions: Studies showed that screening is most likely to be cost-effective in a high TB prevalence population. Our review highlights that to reach the "missing millions"TB programmes should focus on simple, cheaper initial screening tools (i.e., symptom screen and CXR) followed by molecular diagnostic tools (i.e., Xpert ${ }^{\circledR}$ ) among the highest risk groups in the local setting (i.e., PLHIV, urban slums). Programmatic costs greatly impact cost-effectiveness thus future research should provide both fixed and variable costs of screening interventions to improve comparability.

Keywords: Tuberculosis, Economic evaluation, Systematic review, Cost-effectiveness, Tuberculosis in HIV-infected, Tuberculosis control

*Correspondence: azwerlin@uottawa.ca

1 School of Epidemiology and Public Health, University of Ottawa, 600 Peter Morand Cresent, Ottawa, Canada

Full list of author information is available at the end of the article

\begin{abstract}
Introduction
The 2020 Global Tuberculosis (TB) Report noted 3 million undiagnosed TB cases annually worldwide [1]. There is an urgent need to strengthen TB programme efforts to improve TB case detection of these missing millions. These efforts involve scaling up systematic screening programmes targeting household contacts and other
\end{abstract}

(c) The Author(s) 2021. Open Access This article is licensed under a Creative Commons Attribution 4.0 International License, which permits use, sharing, adaptation, distribution and reproduction in any medium or format, as long as you give appropriate credit to the original author(s) and the source, provide a link to the Creative Commons licence, and indicate if changes were made. The images or other third party material in this article are included in the article's Creative Commons licence, unless indicated otherwise in a credit line to the material. If material is not included in the article's Creative Commons licence and your intended use is not permitted by statutory regulation or exceeds the permitted use, you will need to obtain permission directly from the copyright holder. To view a copy of this licence, visit http://creativecommons.org/licenses/by/4.0/. The Creative Commons Public Domain Dedication waiver (http://creativeco mmons.org/publicdomain/zero/1.0/) applies to the data made available in this article, unless otherwise stated in a credit line to the data. 
high-risk groups, including people living with HIV (PLHIV), thereby linking the "missing millions" with diagnosis and treatment. The recent COVID-19 pandemic has dramatically decreased access to TB services and treatment globally, and thus there is an urgent need to get TB programmes back on track to avoid missing even more people who need access to care and treatment.

Passive case finding (PCF) has been the standard for TB diagnosis, and relies on symptomatic individuals selfpresenting to healthcare facilities for diagnosis and treatment [2]. Systematic screening is defined by the WHO as the "systematic identification of people at risk for TB disease, in a predetermined target group, by assessing symptoms and using test, examinations or other procedures that can be applied rapidly" [3]. Active case finding (ACF) is a term for systematic screening of people who do not seek out healthcare services [4] and intensive case finding (ICF) is a term for the regular screening for signs and symptoms of TB among people living with HIV [5]. Systematic screening is a broader term which encompasses all TB screening interventions that actively seek to identify people at risk of TB disease. Screening individuals earlier in their disease course decreases the time during which transmission can occur, with the hopes of reducing future $\mathrm{TB}$ incidence [6].

Individual programmatic components of systematic screening vary by setting, but may include: door-todoor symptom screening, targeted testing of asymptomatic household contacts of persons with TB disease, or screening campaigns among high-risk subpopulations [7]. The algorithms used for systematic screening can involve different screening tools, such as symptom screening or chest X-ray (CXR), and a variety of diagnostic tools including computer-automated detection (CAD) software for X-ray films, GeneXpert MTB/RIF assay $\left(\right.$ Xpert $\left.^{\circledR}\right)$, detection of mycobacterial lipoarabinomannan (LAM) antigen in urine, sputum smear microscopy and inflammatory blood work. The success of screening is dependent on both programme and population factors, such as the underlying prevalence of tuberculosis within a population (i.e., pretest probability of detecting TB). The generalizability of one screening strategy to another community cannot be assumed [8].

PLHIV are a key high-risk group for developing TB disease, accounting for approximately $8 \%$ of all TB cases globally [1]. Clinical risk factors, like diabetes mellitus, [9] and structural risk factors, such as people residing in prisons, [5] also greatly increase the risk of developing TB and have been suggested as high-risk groups to target for screening programmes. Screening programmes typically incur large costs [10] since high-risk groups for TB are often marginalized or living in difficult to access regions [11].
Economic evaluations may help in ensuring that limited resources are used wisely. The current published literature includes systematic reviews of screening interventions and their economic impact but focused on specific populations (i.e., persons experiencing homelessness or incarceration, PLHIV, immigrants or other high-risk groups) [12-17]. These reviews highlight that although systematic screening and active case finding is recommended in high-risk groups, there is a need for clearer guidance on which specific tools and screening algorithms or strategies are cost-effective, essentially highlighting the gap in knowledge that still exists despite WHO's endorsement of systematic screening. Two systematic reviews of ICF among PLHIV showed significant variability across countries and target groups of patients, but highlighted that ICF was cost-saving compared to PCF in high TB/HIV burden countries, though authors noted the lack of standardized methods for cost data collection $[5,17]$. The other reviews of the cost of TB screening were focused on a specific population and setting (i.e., immigrants in low-TB burden settings or contacts in Eastern Europe) [13-15] and recommended systematic screening for high-risk groups, but noted that there was limited data which was heterogeneous and of low quality. Therefore, our objective was to comprehensively synthesize economic evaluations of systematic screening for TB disease to inform a guideline development meeting leading to the updated guidance on TB screening, "WHO consolidated guidelines on tuberculosis. Module 2: screening-systematic screening for tuberculosis disease" [3] (see full list of PICO questions in the Additional file 1). Our study aimed to provide an up-to-date and comprehensive review of the economic evidence for all systematic screening interventions that was not limited to one sub-population, high-risk group, or setting.

\section{Methods \\ Protocol}

We performed a systematic review of the published literature on economic evaluations for TB screening with a focus on high-risk populations of persons with clinical risk factors such as PLHIV, diabetes or other respiratory diseases. We also focused on persons with structural risk factors, defined by the WHO as, "the circumstances in which people are born, grow up, live, work and age," [18] including persons experiencing homelessness or residing in prisons, miners, elderly or indigenous persons. We sought to understand costs, cost-effectiveness, and affordability of screening approaches in key high-risk populations from the health system perspective. We performed this review according to the PRISMA guidelines (see Additional file 1) [19, 20]. 


\section{Information sources}

We searched three online databases: Ovid, EMBASE and Scopus for new studies published within the past ten years (i.e., January 1, 2010 through February 1, 2020). This review was intended to inform an update to the current WHO guidelines. Technologies used for screening and diagnosis of TB have significantly improved in recent years, such as the development of rapid molecular tests for TB including Xpert ${ }^{\circledR}$ MTB/ RIF (Xpert) and other diagnostics that were not available before 2010. A search strategy was developed to identify cost and cost-effectiveness studies of systematic screening in high-risk groups. We reviewed citations of all eligible articles, guidelines, and reviews for additional studies (see Additional file 1 for search terms).

\section{Study selection}

Studies were included if they evaluated any type of systematic screening activities among persons with clinical or structural risk factors and included costs. Our search terms were designed to broadly capture any economic evaluations or studies including costs and an outcome, such as disability-adjusted life years (DALYs) or qualityadjusted life years (QALYs) and was not limited to costeffectiveness or cost-utility analyses. Studies including utilities, such as DALYs, without costs were not included. Relevant studies were identified through electronic searches of the online databases, and duplicates were removed. Articles were excluded if they did not evaluate screening activities, or were reviews, letters, or opinion pieces.

\section{Data collection}

Articles were excluded if they only screened for latent TB infection, did not report costs per person screened or diagnosed or did not report the costs for the screening intervention separately from standard care (i.e., PCF). Studies were excluded if they evaluated screening activities in the general population, among contacts or children, since these groups were included in a separate manuscript (accepted, in press). Full text review was done independently by two reviewers (HA and $\mathrm{BE}$ ) on remaining articles that met predetermined inclusion criteria, with all disagreements resolved by discussion with a third reviewer (AZ). No language filter was applied. Assessment of the quality of each economic evaluation and study quality was guided by the Consensus Health Economic Criteria (CHEC) [21, 22] and CHEERS checklist, respectively (see Additional file 1: Tables S1 and S2) [21].

\section{Data extraction}

The study data elements extracted from each study included: primary research question, country and setting, year of study, patient population, clinical setting, type of intervention, comparison diagnostic scenarios, economic analysis perspective, analytic time horizon, type of economic evaluation, source of costing, primary and secondary outcome measures, type of model, sensitivity and uncertainty analyses performed and willingness-topay (WTP) threshold. The WHO's World Health Report guidelines on Choosing Interventions that are Cost-Effective (CHOICE) are the most commonly referenced WTP threshold among cost-effectiveness studies, particularly in low- and middle-income countries (LMICs) and are typically based on 1-3 times the country gross domestic product (GDP) per capita [23]. However, the use of these GDP-based thresholds has been challenged by many experts, the thresholds are considered overly simplistic and too easily attained when an intervention is effective [24]. Another key criticism of GDP-based WTP thresholds is the lack of their value and usefulness in assessing the trade-offs that decision-makers face in allocating limited healthcare resources [24]. Although the WHO no longer endorses GDP-based WTP thresholds [25], the challenge remains for clinicians, programme managers and researchers to determine the best metric for assessing value and reporting outcomes for cost-effectiveness and affordability of healthcare interventions. For the purposes of this review and to enable comparisons across currently published studies, we have included the GDPbased WTP thresholds used by study authors. However, we support the need for better decision-making tools for resource allocation in the local context, bearing in mind opportunity costs and the burden of disease.

Model parameters were extracted including epidemiologic, treatment and outcome parameters. Key outcomes included: cost per patient diagnosed, and incremental cost-effectiveness ratio (ICER) per utility measure (e.g., DALY averted). Costs are presented in United States Dollars (USD) adjusted to 2019 [27, 28]. Given the heterogeneity of study setting, year and type of screening strategies employed, there was no plan to calculate global estimates or pool data.

\section{Results \\ Study selection}

We identified a total of 3481 articles through database searching (Fig. 1). After duplicate removal, we screened 2318 citations by title and abstract for inclusion. Of these, we assessed 145 full-text publications against our inclusion criteria and excluded 118 publications. Exclusions were mainly due to the wrong intervention or no 


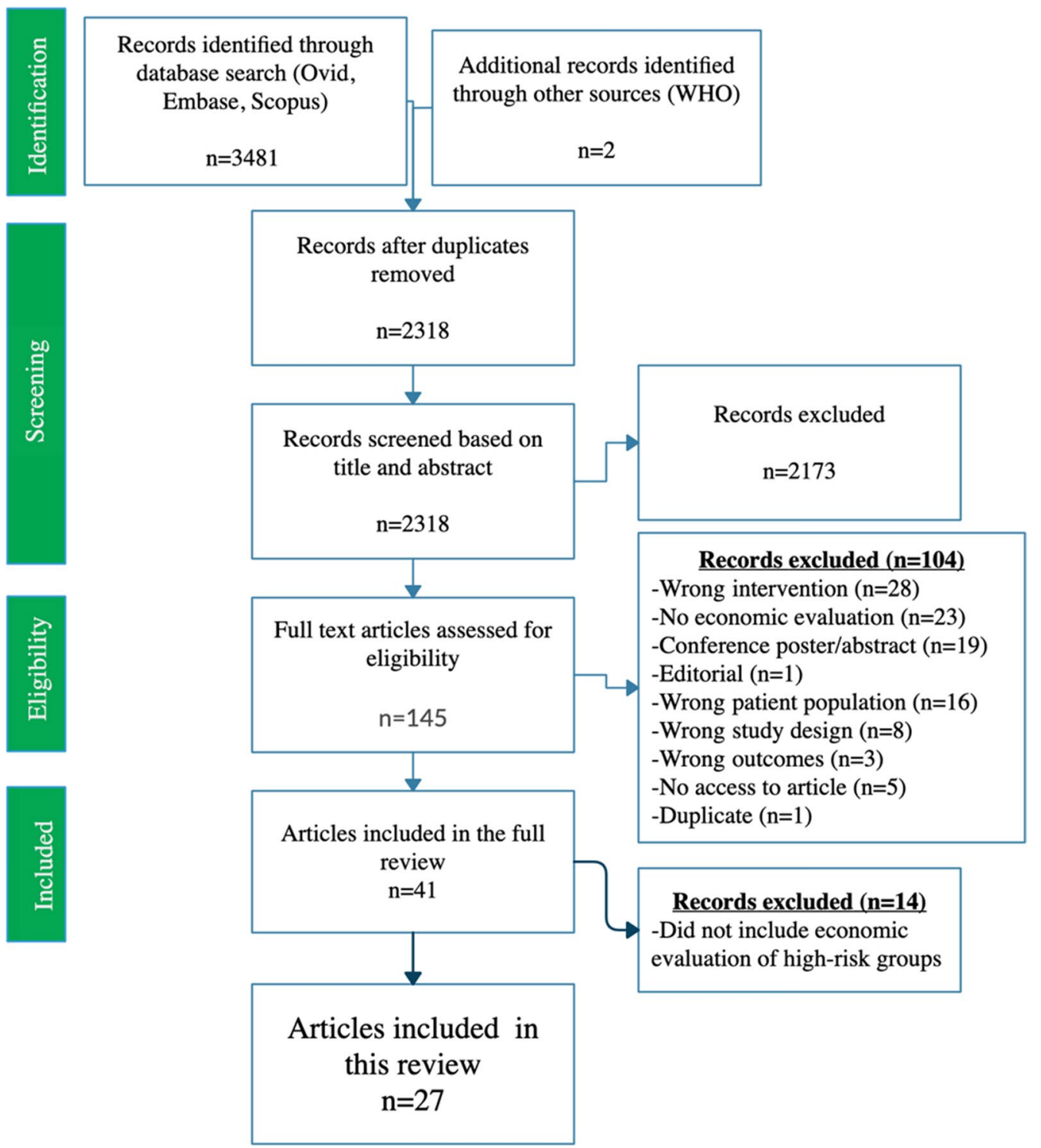

Fig. 1 PRISMA diagram

economic evaluation. A total of 27 studies were identified for inclusion in the review [11, 29-53].

\section{Study characteristics}

Study characteristics for the 27 included studies are summarized in Tables 1 and 2; 19/27 (70\%) of studies were conducted in high TB burden countries. Some studies included stratified analyses among multiple high-risk populations and thus contributed results to multiple categories (i.e., clinical and/or structural risk factors). Seventeen studies included persons with clinical risk factors; [32, 34, 38] fourteen among PLHIV, the majority of which (12/14) were conducted in Sub-Saharan Africa
(SSA) [29-32, 37, 39-42, 44, 45, 49, 52, 53]. Thirteen studies included persons with structural risk factors (i.e., migrants, persons experiencing homelessness, or miners) and were from a range of countries such as Belgium, Cambodia, China, Russia, South Africa, and Zimbabwe $[11,32,33,35,36,38,43,46-48,51,54]$.

\section{Study findings}

We present the study findings stratified by high-risk subgroup, including persons with clinical and structural risk factors and PLHIV, as well as by screening tool used below. 


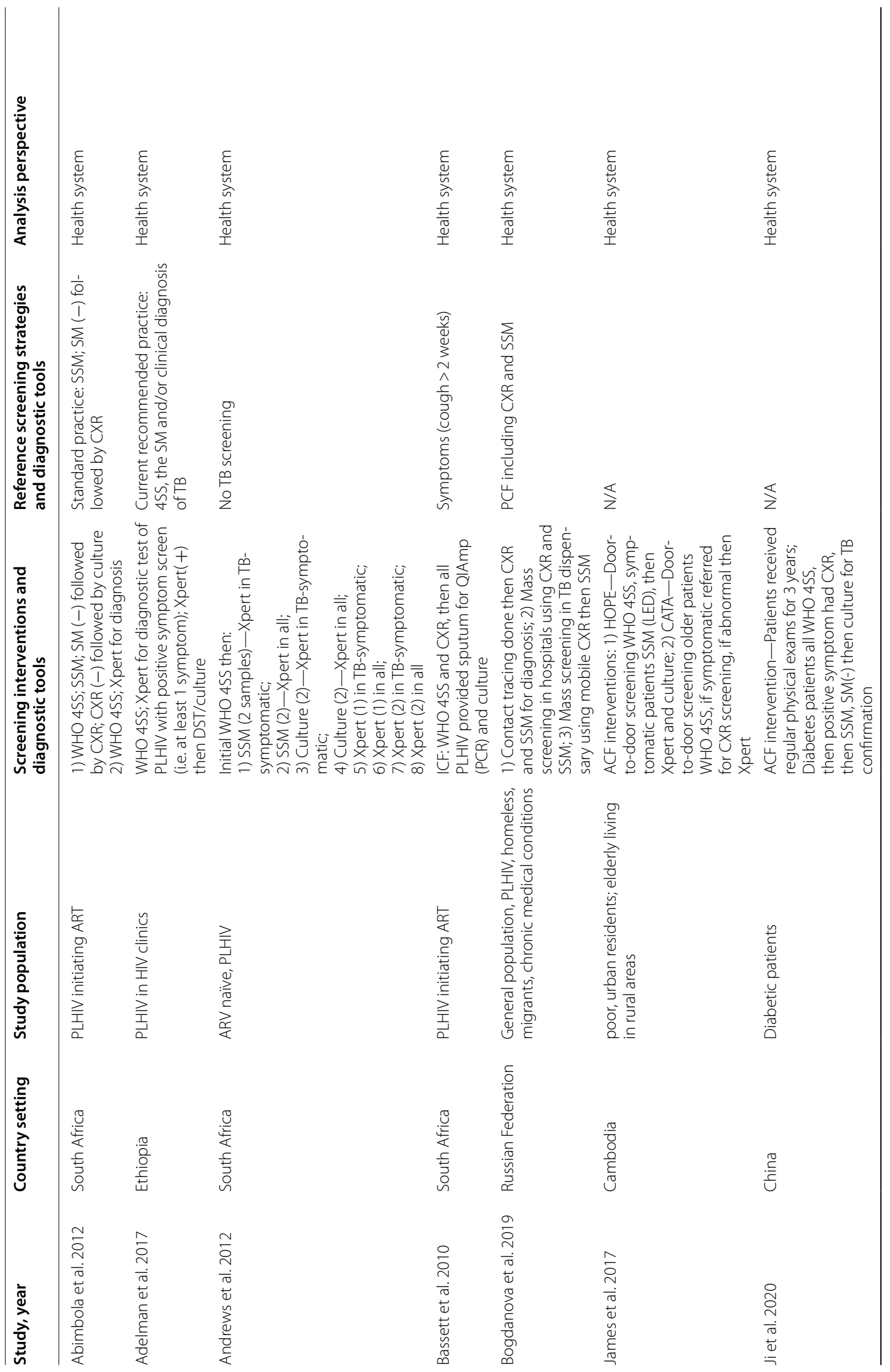




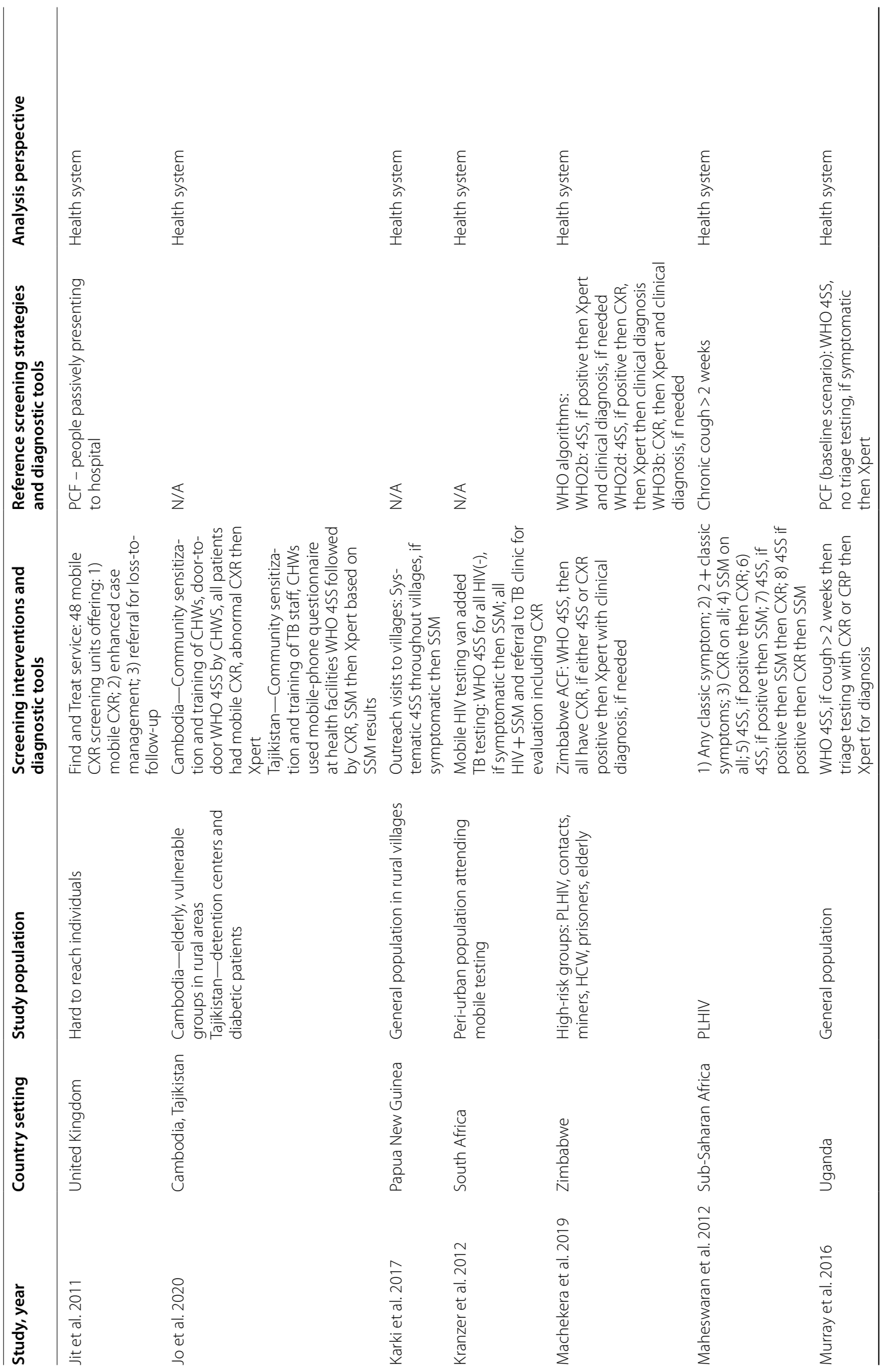




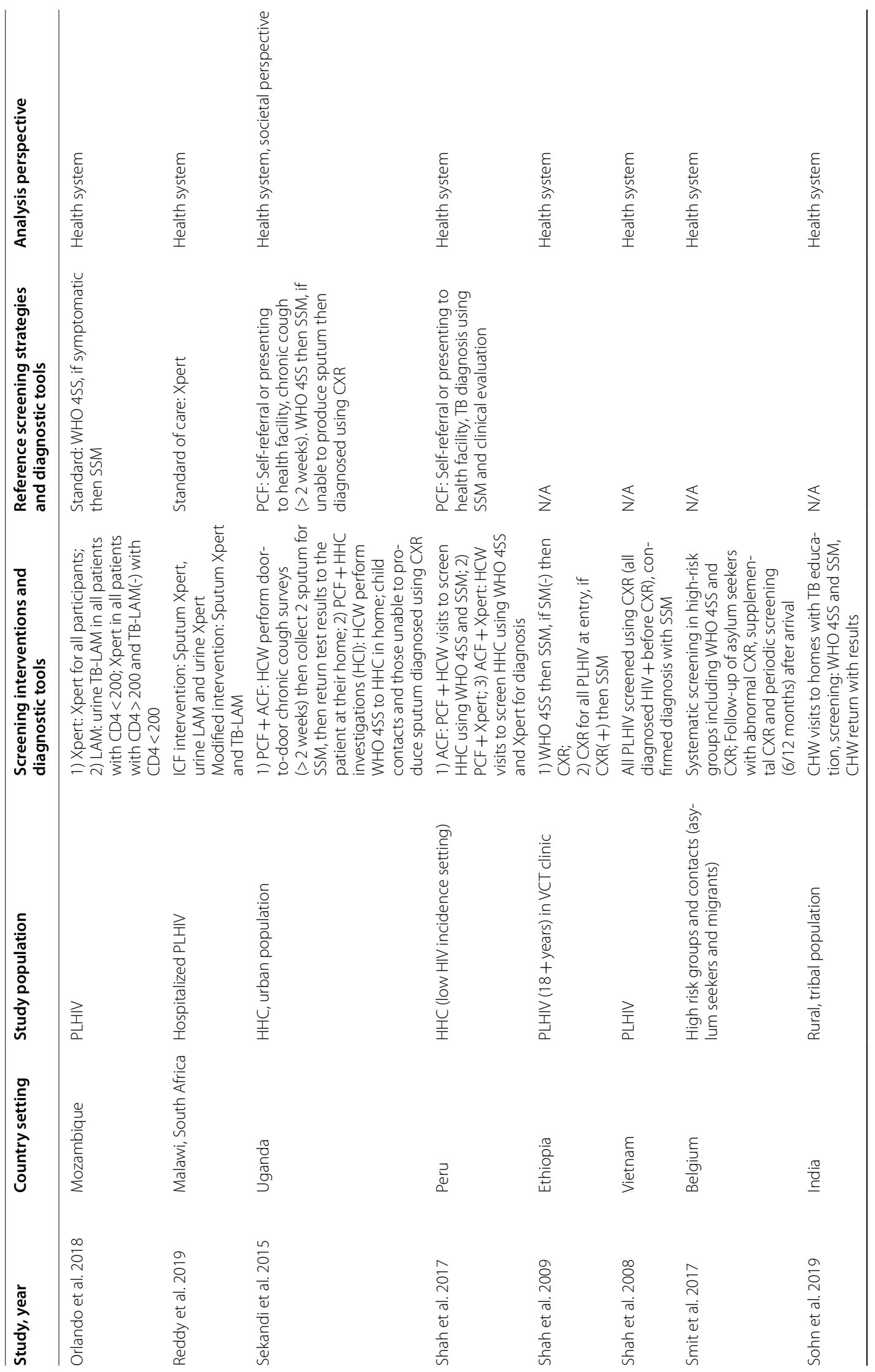




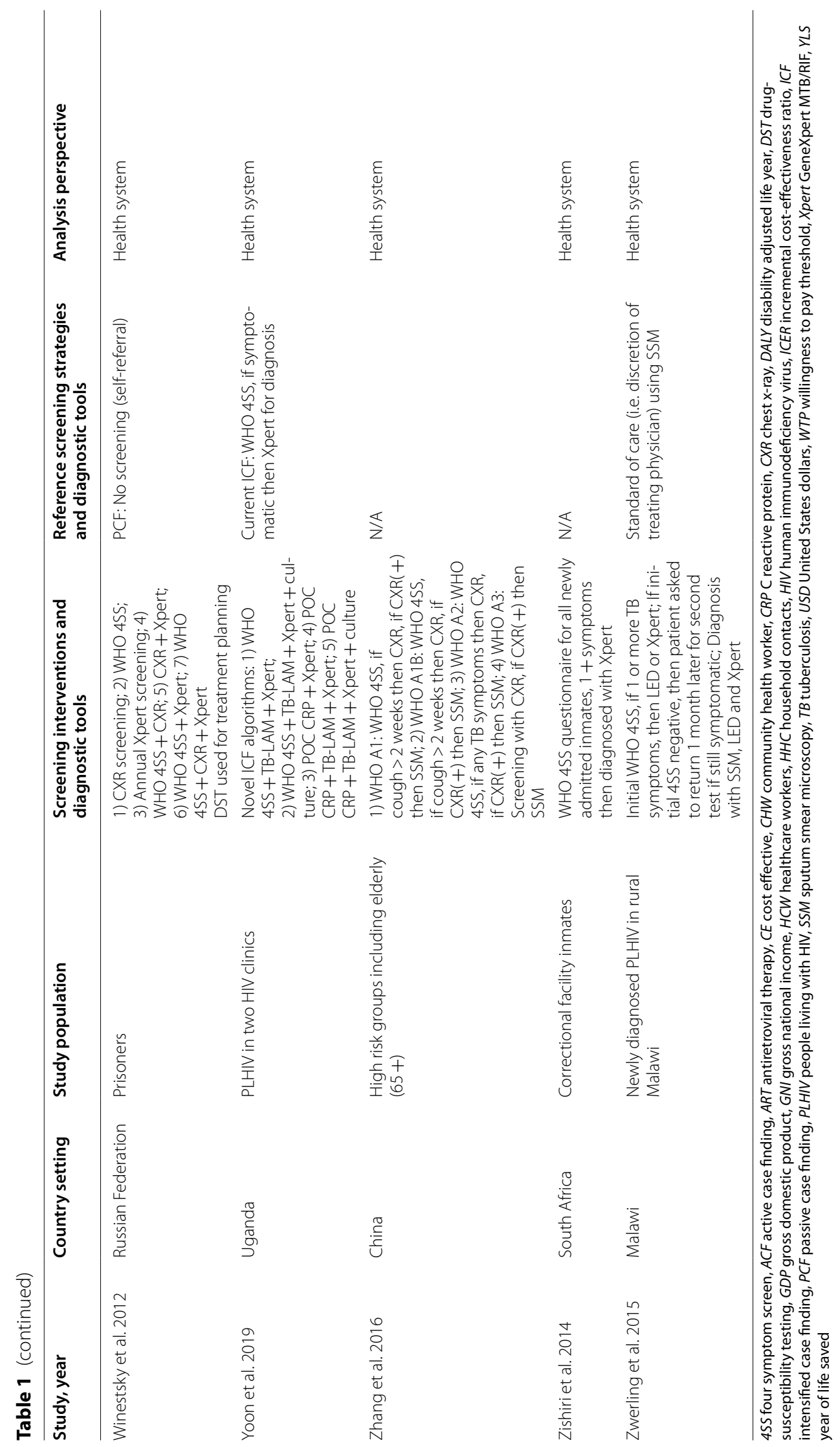




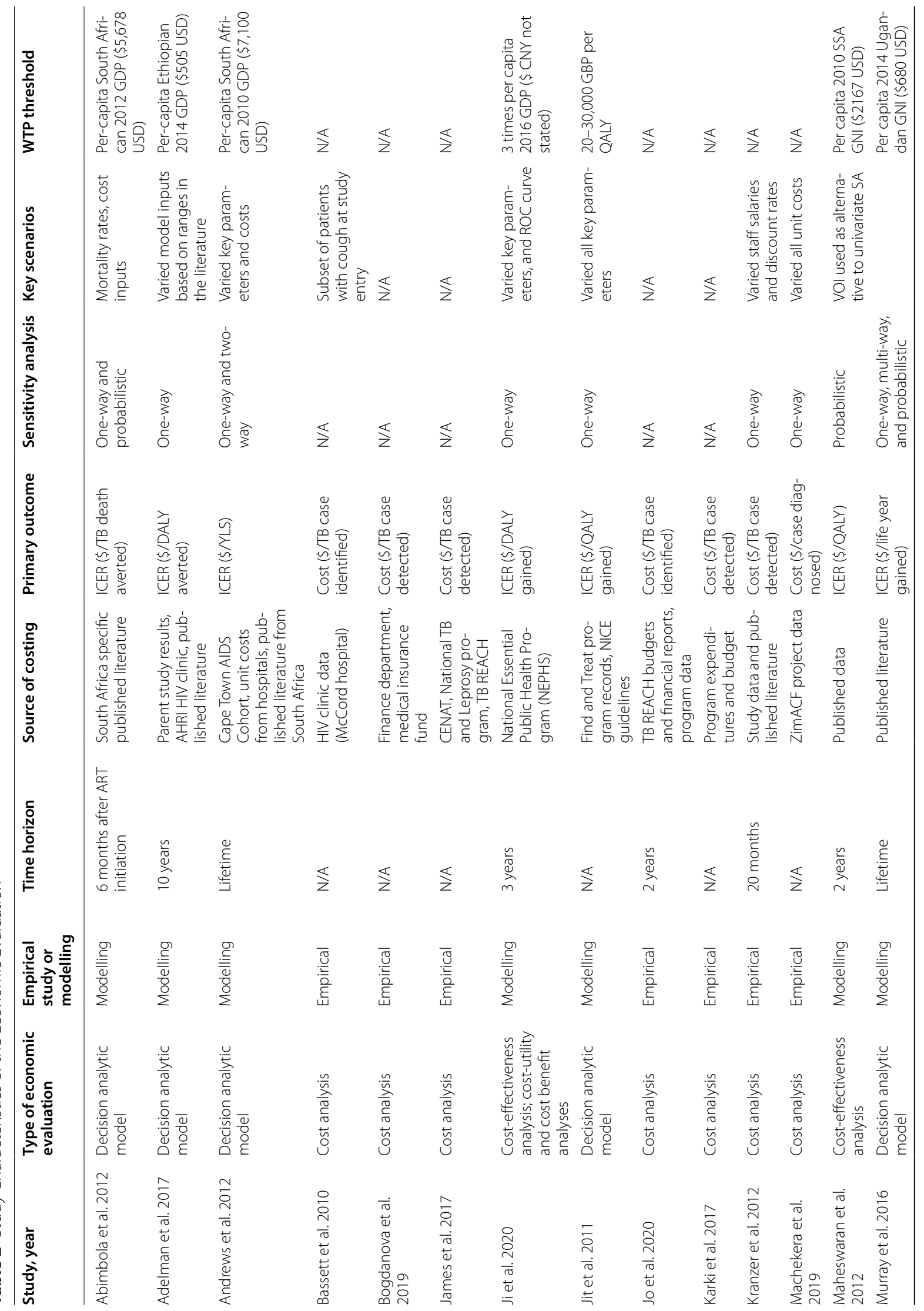




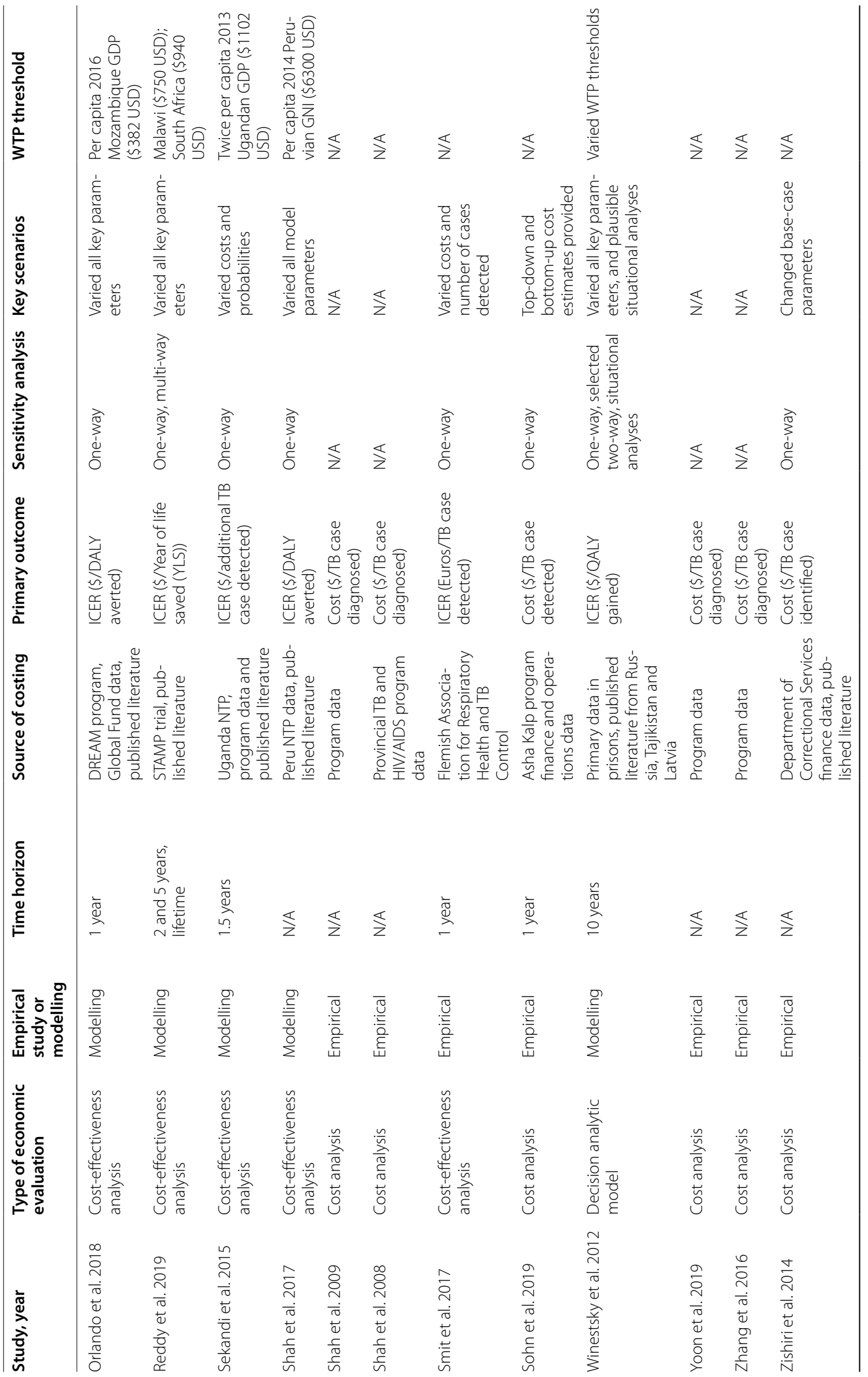




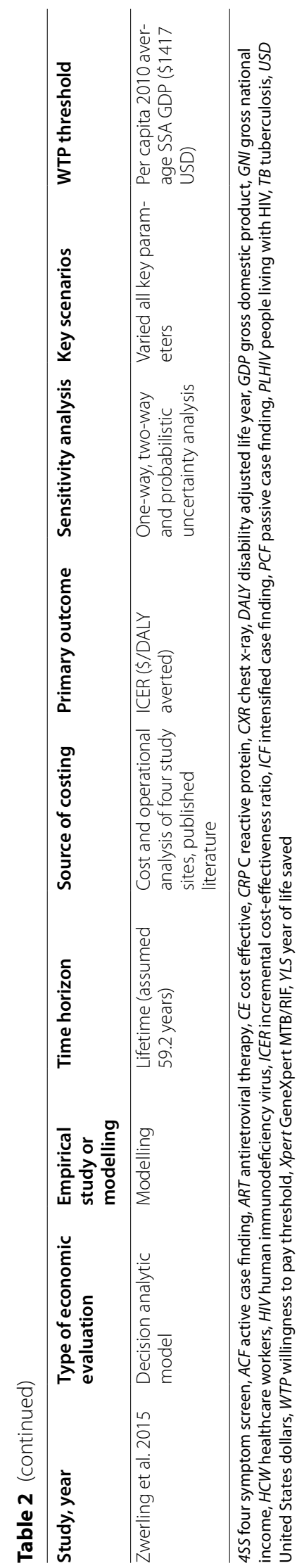




\section{Persons with clinical risk factors}

Three studies provided cost-effectiveness data for individuals with the following clinical risk factors: diabetes mellitus, chronic respiratory disease and fibrotic lesions (Table 3) [32, 34, 38].

\section{Diabetes and other medical co-morbidities}

Bogdanova et al. assessed screening with chest X-ray (CXR) in Russia, and stratified results among diabetic patients as well as persons with other medical comorbidities (i.e., respiratory disease, gastro-intestinal, or fibrotic chest lesions); [32] reported costs ranged from US\$11,648 to $\$ 105,754$ per TB case diagnosed [32]. In Zimbabwe, Machekera et al. found that screening diabetic patients with CXR followed by Xpert ${ }^{\circledR}$ cost US $\$ 2191$ per person diagnosed with TB [38]. Ji et al. reported that routine screening with CXR among Chinese patients with diabetes was considered highly cost-effective, as compared to PCF, with an ICER of US\$288 per DALY averted [34]. Machekera et al. included personnel and laboratory costs and had a large number needed to screen which drove up the cost per person diagnosed, while Ji et al. did not include the overhead costs for diabetic patient care.

\section{People living with HIV (PLHIV)}

There was significant heterogeneity of screening and diagnostic tools used among the 14 studies reporting the cost and cost-effectiveness of programmes in PLHIV (Table 4). Studies often considered multiple diagnostic algorithms or tools. Seven studies used molecular rapid diagnostic tests (i.e., Xpert ${ }^{\circledR}$ MTB/RIF) as an initial screening tool; [29-31, 40-42, 49, 52, 53] six studies used CXR to screen PLHIV in the outpatient setting; [31, 32, $39,40,44,45]$ two studies used C-reactive protein (CRP) to screen; [40, 49] and one study used sputum smear microscopy (SSM) alone for screening [37].

\section{Screening PLHIV with molecular rapid diagnostics}

All seven studies that assessed screening in PLHIV using $\mathrm{Xpert}^{\circledR} \mathrm{MTB} / \mathrm{RIF}$ as an initial test were conducted in Sub-Saharan Africa, with 6/7 concluding screening was cost-effective. Two studies among PLHIV in South Africa conducted initial WHO-recommended four symptom screen (W4SS), including screening for cough of any duration, weight loss, fever or night sweats [55], followed by screening with Xpert ${ }^{\circledR}$ and found screening to be costeffective with ICERs of US $\$ 324$ per additional TB patient diagnosed and US $\$ 48,542$ per TB death averted [29, 31]. Andrews et al. used two Xperts ${ }^{\circledR}$ to screen all PLHIV at a clinic in South Africa which was found to be costeffective with an ICER of $\$ 4,096$ per year of life saved (YLS) (WTP threshold: $\$ 7100$ per YLS). [30] Among
PLHIV initiating anti-retroviral therapy (ART), a combination of HIV treatment medications, in Mozambique, Orlando et al. showed that W4SS followed by screening with either Xpert ${ }^{\circledR}$ alone or Xpert ${ }^{\circledR}$ and lateral flow urine lipoarabinomannan assay (LF-LAM) was cost-effective, compared to W4SS and SSM, with ICERs ranging from US $\$ 37$ to $\$ 51$ per DALY averted [41]. Reddy et al. modeled the impact of a screening intervention with TBLAM, urine and sputum Xpert ${ }^{\circledR}$ in hospitalized PLHIV, regardless of symptoms, in Malawi and South Africa [42]. The intervention was cost-effective, compared to sputum Xpert ${ }^{\circledR}$ alone (standard of care), with reported ICERs of US\$450 and US\$840 per YLS in Malawi and South Africa (WTP threshold: $\$ 750$ and $\$ 940$ per YLS, respectively).

Zwerling et al. found a randomized controlled trial using point of care (POC) Xpert ${ }^{\circledR}$ to screen PLHIV in rural Malawi was not cost-effective, due to low-test volumes (i.e., 50 tests/year, ICER of US $\$ 1980$ per DALY averted, Uncertainty Range (UR): \$1544-\$3552). Zwerling et al. showed that Xpert ${ }^{\circledR}$ could be cost-effective at higher-test volumes (1000 tests/year, ICERs of US\$398 per DALY averted, UR: \$80-\$1682) [WTP threshold: $3 \times$ GDP per capita of Malawi (US\$254)].

\section{Screening PLHIV with CXR}

Four of the six studies (67\%) reporting on the use of CXR to screen PLHIV were conducted in SSA. The cost per person diagnosed with TB ranged from US $\$ 106$ to $\$ 570$ [31, 32, 44, 45]. Murray et al. found that community screening for cough followed by CXR in Uganda was cost-effective with an ICER of US\$536 per year of life gained (YLG) (UR: \$176-\$2514) [40] Mahesawaran et al. showed the W4SS followed by CXR in SSA was cost-effective with an ICER of US\$6245 per QALY (UR: \$6245-\$19,581) [WTP threshold: $3 \times$ GNI per capita (US\$2167)] [39].

\section{Screening PLHIV with CRP}

Among ART-naïve HIV clinic patients in Uganda, Yoon et al. found POC CRP followed by Xpert ${ }^{\circledR}$ for diagnosis [49] algorithms were cost-saving compared to W4SS followed by Xpert ${ }^{\circledR}$ (standard of care), ranging from US $\$ 69$ to $\$ 92$ per TB patient diagnosed. Murray et al. showed that CRP was cost-effective as a triage test in PLHIV in Uganda, compared to Xpert ${ }^{\circledR}$, with an ICER of $\$ 517$ per YLG (UR: \$176-\$2514) [WTP threshold: Ugandan per capita GDP (US\$609)]. [40].

\section{Screening among PLHIV using SSM}

Kranzer et al. performed an intervention of adding TB symptom screening and SSM to an existing mobile HIV testing clinic in South Africa, [37] with an average cost of US\$762 per TB case diagnosed [37]. 


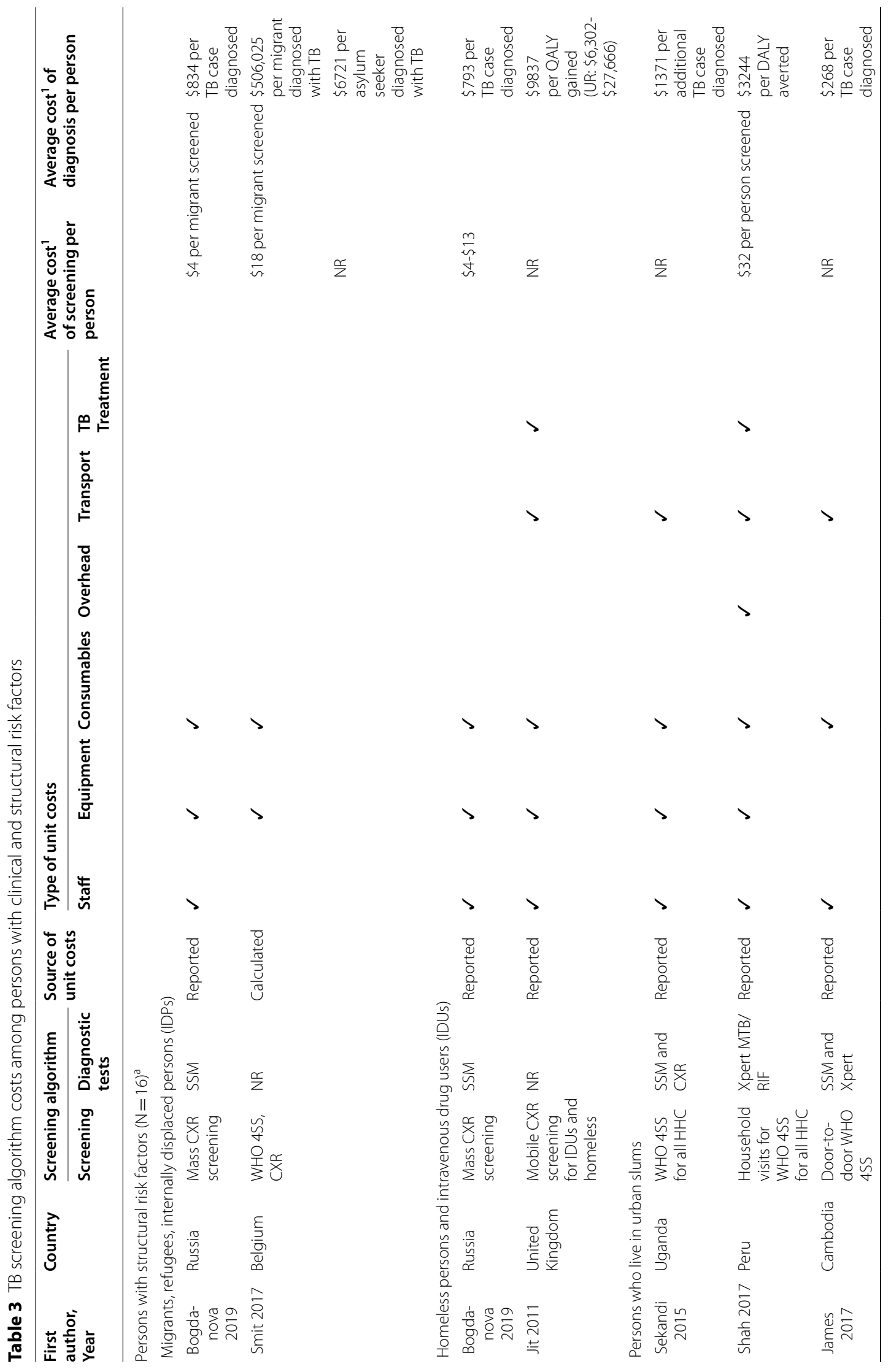




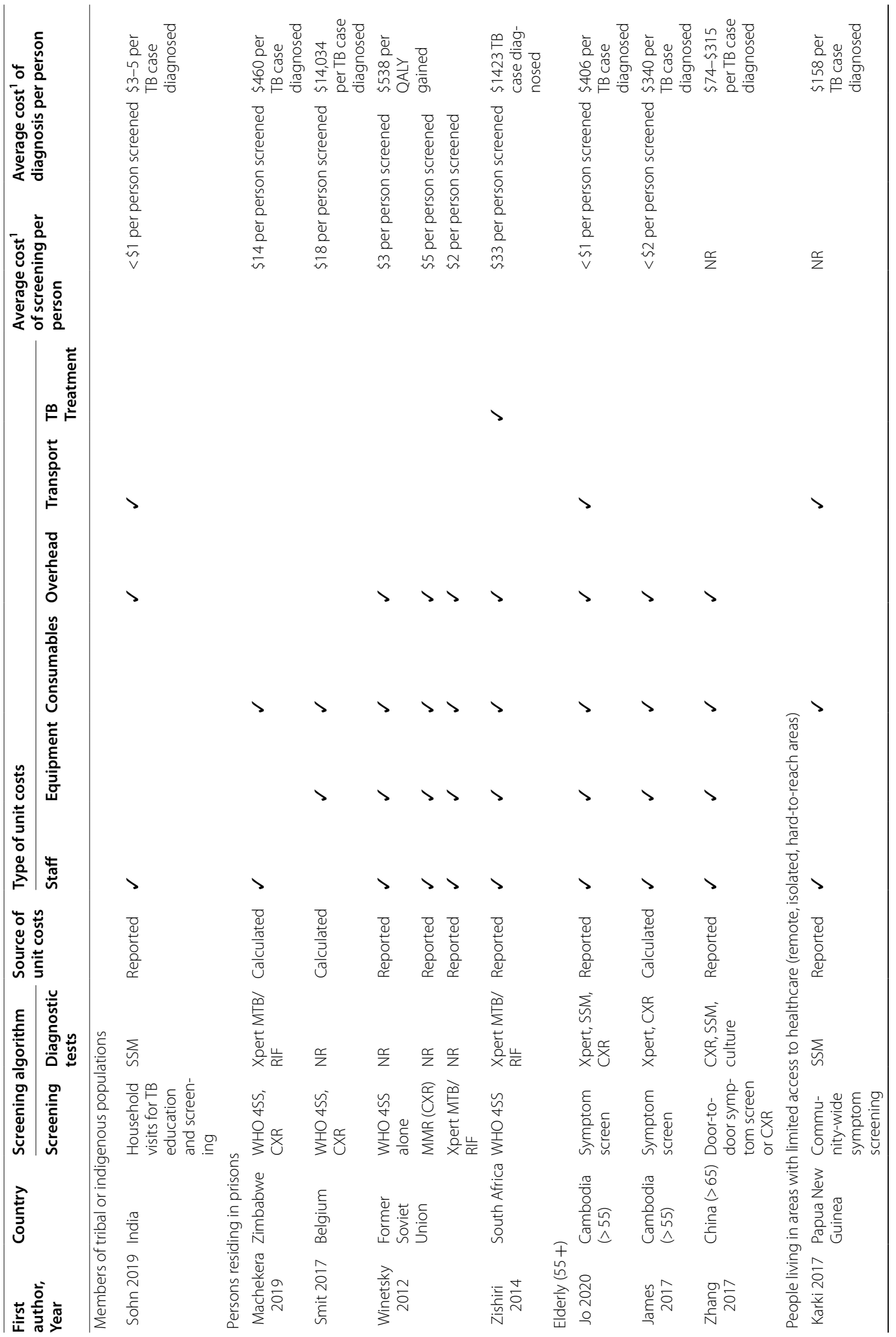




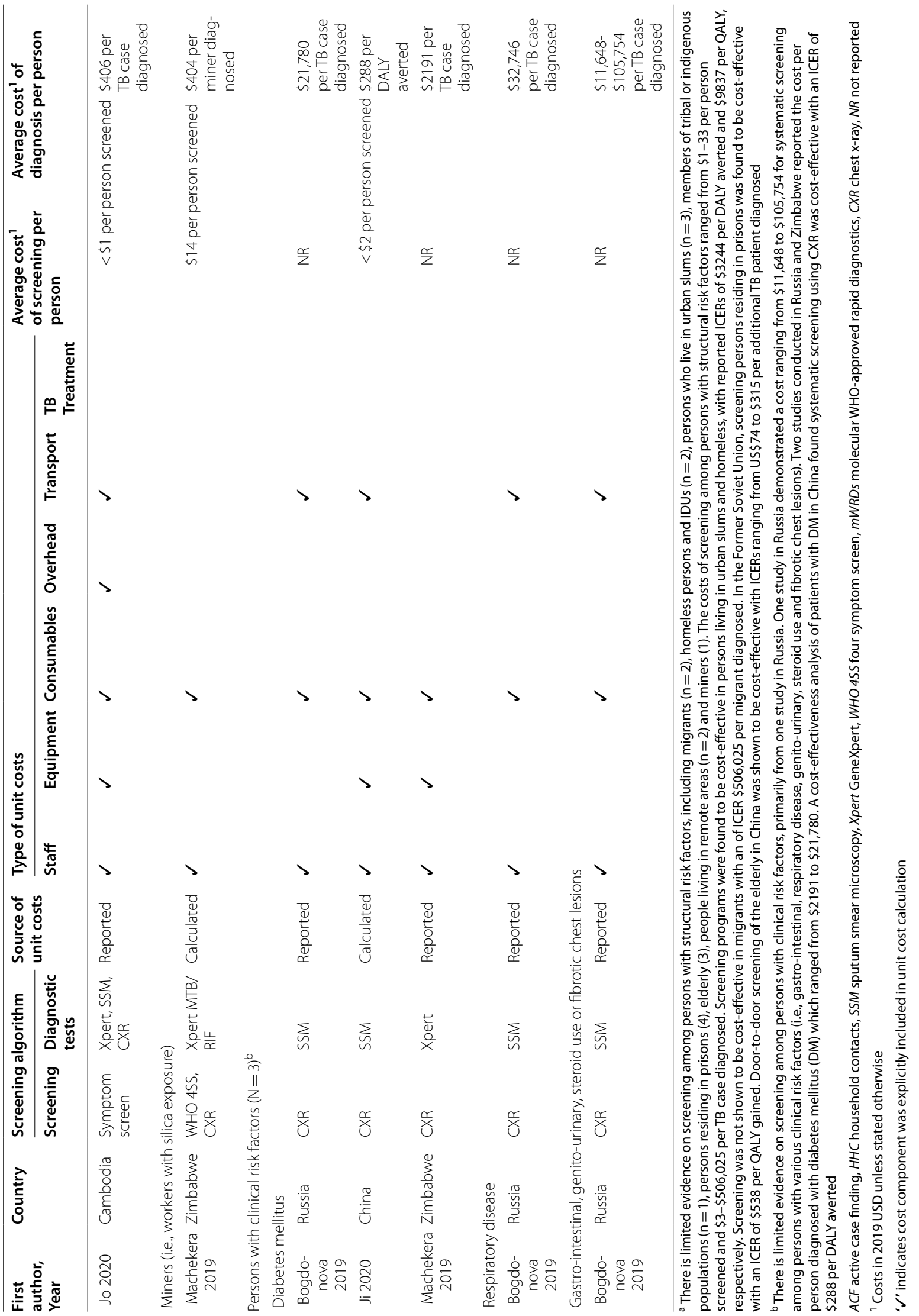




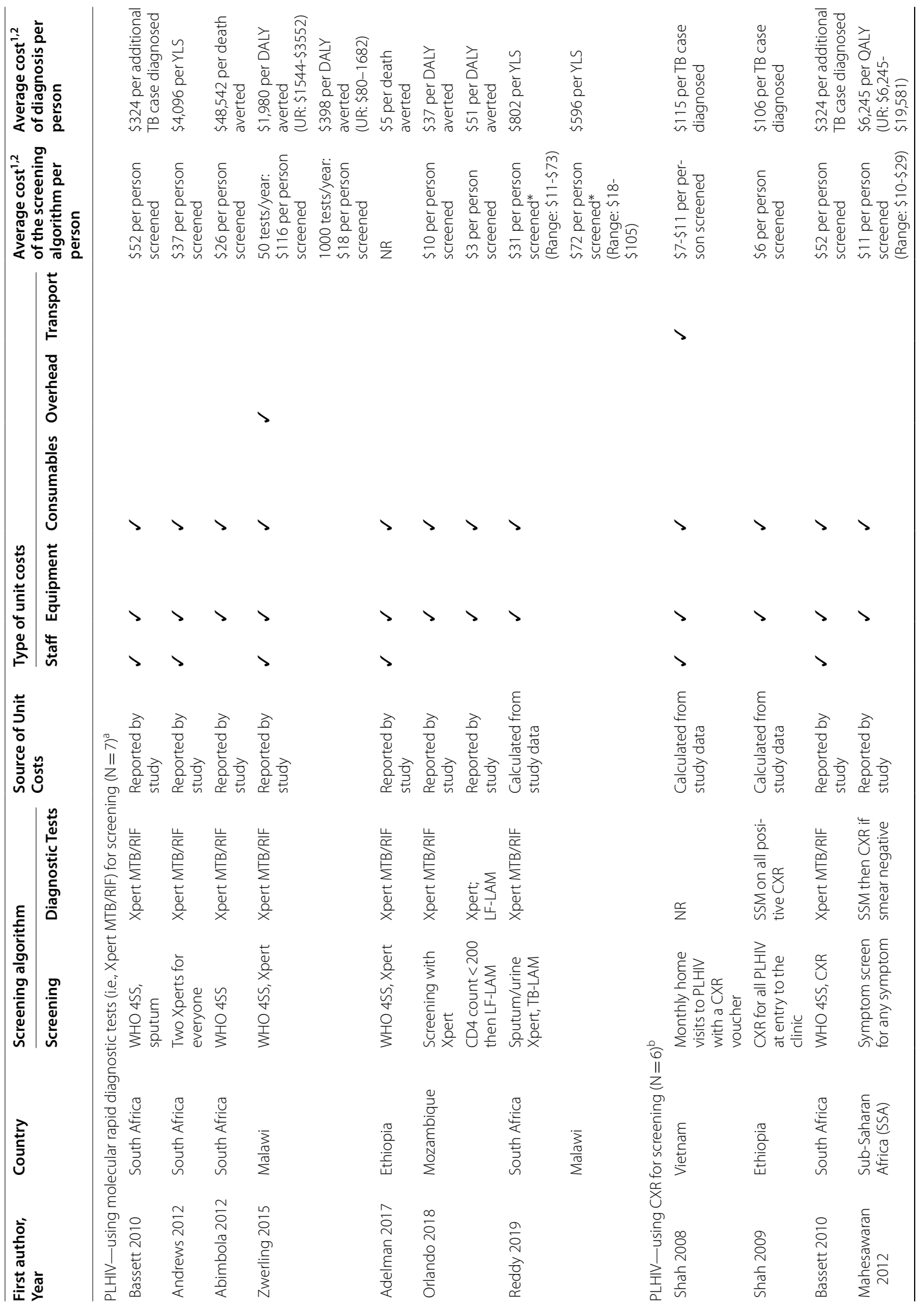




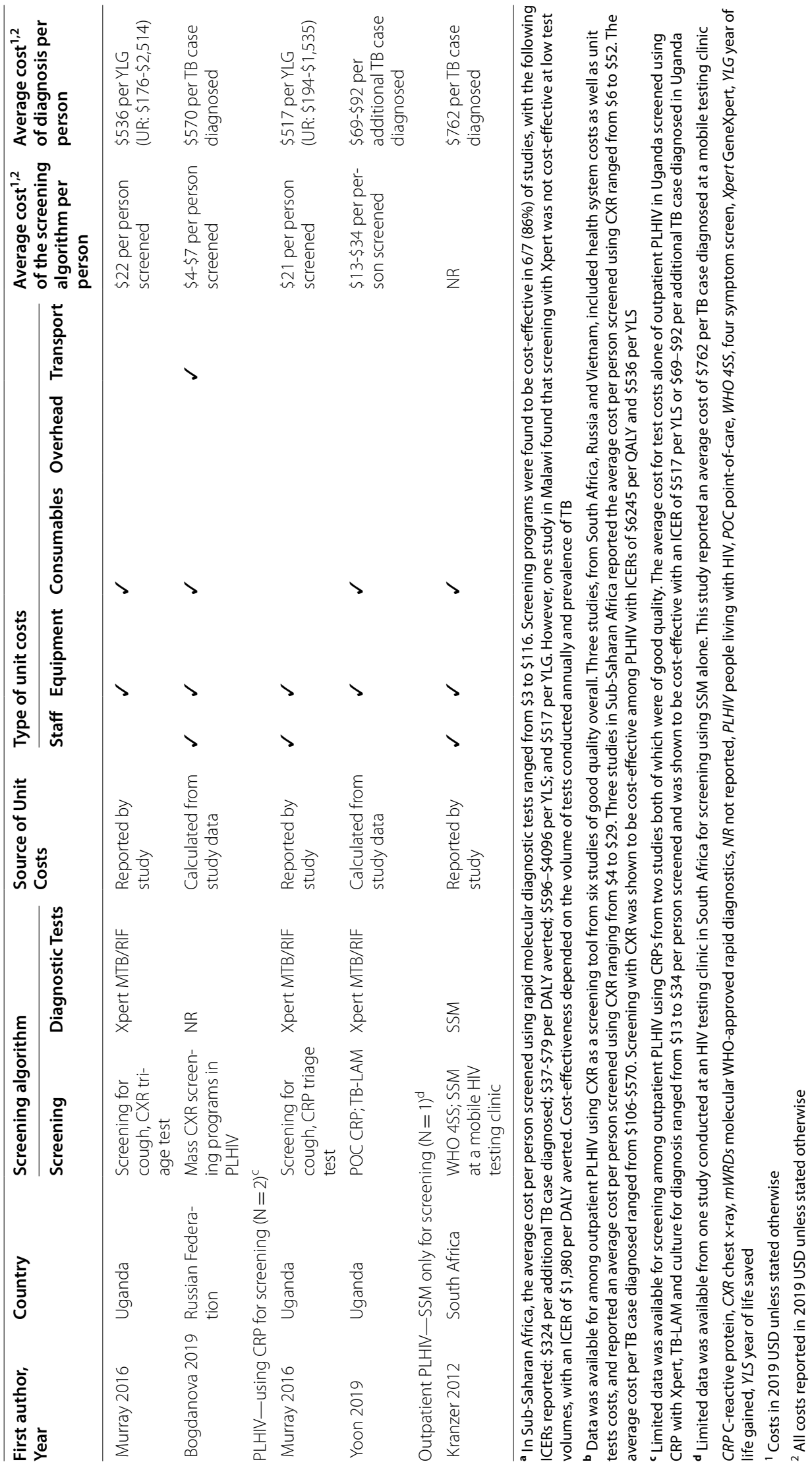




\section{Persons with structural risk factors}

Thirteen studies provided cost effectiveness data for individuals with structural risk factors including (Table 3): migrants, [32, 47] persons experiencing homeless, [11, 32] persons who live in urban slums, [33, 43, 46] members of indigenous populations, [54] persons residing in prison, $[38,47,48,51]$ elderly, $[33,35,50]$ people living in remote areas, $[35,36]$ and miners [38].

\section{Migrants, persons experiencing homelessness and intravenous drug users (IDUs)}

Bogdanova et al. assessed the cost of screening with CXR in Russia in multiple subgroups and found a cost of US\$834 and US\$793 per migrant and homeless person diagnosed with TB, respectively [32]. Jit et al. demonstrated that a programme using mobile screening vans among persons experiencing homelessness and IDUs in London, United Kingdom was cost-effective, compared to PCF, with an ICER of USD \$9837 (UR: \$6301-\$28,666) per QALY gained (WTP threshold: $£ 20,000-£ 30,000$ per QALY gained set by NICE standards) [11]. However, in Belgium, Smit et al. showed CXR screening among migrants from high TB incidence countries was not costeffective, compared with PCF, with an ICER of $\$ 506,025$ (95\% UR: \$90,686-\$2,040,006) per additional TB case diagnosed [47].

\section{Persons who live in urban slums}

Three studies examined door-to-door screening interventions in urban slums. James et al. reported that doorto-door symptom screening in Cambodian slums using CXR followed by Xpert ${ }^{\circledR}$ for diagnosis cost US $\$ 268$ per TB case diagnosed [33]. Shah et al. demonstrated that household visits to screen all contacts of persons with $\mathrm{TB}$ in an urban slum in Lima, Peru was cost-effective, compared to PCF, with an ICER of US\$3244 per DALY averted [WTP threshold: 2014 per capita GNI for Peru (US\$6360)]. [46] However, Sekandi et al. found that doorto-door symptom screening of all household contacts, followed by SSM and CXR in urban Uganda, was not cost-effective compared to PCF with an ICER of US\$1371 per additional TB case diagnosed [WTP threshold: twice the 2012 Ugandan per capita GDP (US\$551)]. [43].

\section{Members of indigenous populations}

Sohn et al. reported a cost of US $\$ 3-\$ 5$ per person detected with TB for a programme of home visits by community health workers (CHWs) to indigenous persons in rural India. This study included the costs for CHWs time to travel to homes, conduct screening and diagnostic visits, provide directly observed therapy (DOT) and care, as well as the laboratory and administrative services, [54] but did not account for routine TB care and medications provided by the Indian government.

\section{Persons residing in prisons}

Four studies provided direct evidence for the costs of screening among persons residing in prisons $[38,47,48$, 51]. Zishiri et al. found W4SS followed by Xpert ${ }^{\circledR}$ for all persons residing in prison in South Africa cost US\$1423 per person diagnosed. [51] While in Zimbabwe, Machekera et al. reported that an intervention of W4SS and CXR followed by Xpert ${ }^{\circledR}$ cost US $\$ 460$ per person diagnosed [38]. Winetsky et al. performed a dynamic transmission model of $\mathrm{TB}$ among persons residing in prisons in the Former Soviet Union and demonstrated that annual screening with Xpert ${ }^{\circledR}$ was cost-effective compared with mass CXR screening, with an ICER of US\$538 per QALY gained [WTP threshold: per capita GDP of Tajikistan (US\$1900)]. [48] Smit et al. demonstrated that systematic screening of persons residing in prisons using W4SS followed by CXR in Belgium was cost-effective, compared to PCF, with an ICER of US\$14,034 (95\% UR: \$10,898$\$ 18,033)$ per additional case of TB detected. [47].

\section{Elderly}

Two studies were conducted in Cambodia of door-todoor screening using the W4SS followed by CXR and $\mathrm{Xpert}^{\circledR}$ for diagnosis among the elderly $(55+)$, and reported average costs ranging from US\$340 to $\$ 406$ per person diagnosed with TB [33, 35]. Zhang et al. found that door-to-door symptom screening and CXR among the elderly in China $(65+)$ had costs ranging from US\$74-\$315 per TB case diagnosed [50]. Among elderly persons in China, additional risk factors (i.e., male, tobacco use or close TB contact) were associated with higher average costs per patient diagnosed [50].

\section{People living in remote areas}

In Papua New Guinea, Karki et al. reported that an intervention using SSM to screen all villagers with symptoms of TB cost US $\$ 158$ per person detected with TB. [36] A second intervention, conducted by Jo et al. in remote Cambodia, included house-to-house symptom screening followed by mobile CXR and reported a cost of US $\$ 406$ per person diagnosed with TB [35].

\section{Miners}

There was limited evidence for screening in miners from one study in Zimbabwe. [38] Machekera et al. demonstrated that a screening algorithm of CXR followed by Xpert $^{\circledR}$ among those with positive CXR was cost saving, with a cost of US\$404 compared to US\$576 per person 
diagnosed with an algorithm screening everyone with W4SS and CXR.

\section{Discussion}

Our review of the published literature identified 27 studies of systematic screening among high-risk groups for $\mathrm{TB}$, such as PLHIV, miners, persons residing in prisons, and the elderly. Our review found that systematic screening approaches are most likely to be cost-effective in settings and or populations with high prevalence of TB, such as PLHIV, persons residing in prisons and urban slum dwellers. Studies demonstrated that initial screening with more costly diagnostic tests was not cost-effective in high-risk groups, except among PLHIV. Simple, inexpensive initial screening methods (i.e., W4SS or CXR) followed by molecular diagnostic tools (i.e., Xpert ${ }^{\circledR}$ ) among the highest risk groups in the local setting are the most cost-effective approaches to systematic screening. In high TB prevalence settings, door-to-door symptom screening in densely populated areas (i.e., slums), was generally shown to be cost-effective. However, mobile CXR units were not cost-effective due to high programmatic costs, particularly when interventions were targeting hard to reach populations (i.e., persons experiencing homelessness or IDUs). There was limited evidence identified for each high-risk group included in this review, thus caution should be used when extrapolating from a small number of studies.

Our review found the most evidence for cost-effectiveness of screening programmes among PLHIV. Despite varying screening strategies (i.e., W4SS, CRP, CXR, Xpert $^{\circledR}$ MTB/RIF) and patient settings (i.e., in-patient or outpatient clinic), the majority $9 / 10$ (90\%) of PLHIV studies that calculated an ICER found screening interventions to be cost-effective among PLHIV using an author determined WTP threshold. Key drivers of costs among PLHIV included: annual test volume and diagnostic test costs, [52] underlying prevalence of TB and HIV, [42, 52] clinic setting, [32, 44, 45] and programmatic costs included (i.e., transportation, mobile van units, or staffing costs) [31, 32, 55]. In sensitivity analyses of lowTB prevalence settings among PLHIV, screening strategies with simple tools, such as W4SS and SSM, were cost-saving compared to more expensive tools such as CXR or Xpert ${ }^{\circledR}$, [39] highlighting the importance of the local setting.

Screening interventions in high TB prevalence settings, such as urban slums or among persons residing in prisons, increased identification and diagnosis of people with TB and were shown to be cost-effective [46, 48]. Equally important was ensuring proper follow-up to avoid treatment failure or loss post-screening [44]. However, among groups with other medical conditions, high programmatic screening costs, coupled with low TB prevalence meant screening interventions were not costeffective, but was limited to two studies [32, 38].

Systematic screening is an expensive undertaking, particularly compared to PCF, when it involves mobilizing staff to go into the community. Evidence from this review suggests that community-based interventions [43] had higher costs compared to systematic screening programmes targeting persons presenting to healthcare facilities [35]. The use of artificial intelligence (AI) to inform screening tools through the use of CAD software is an exciting prospect for improving the efficiency and affordability of screening from standard CXR. However, our review did not find any studies which used CAD software in the context of a systematic TB screening programme. Indirect evidence, not presented in this review, has shown that the unit costs for each CXR read with CAD software are likely to be small but require significant investment in equipment and maintenance costs as well as the purchase of CAD software. These new technologies are still being evaluated in many programmes and highlight the need for costing and cost-effectiveness studies to inform their use in the programmatic setting.

The WTP thresholds, which are noted a priori by study authors and based on WHO recommendations, determine whether a given ICER is considered cost-effective. Among the papers reviewed in this analysis, many used either a country's GDP, or twice the GDP, as the WTP threshold, however there was significant heterogeneity due to the range of country settings. This variability makes comparisons challenging, particularly since setting a higher threshold increases the likelihood of an intervention being considered cost-effective. Careful attention should be paid to the WTP threshold employed by study authors when interpreting cost-effectiveness. Furthermore, there are concerns about using GDP per capita as the basis of determining cost-effectiveness, particularly in LMICs where there are more stringent resources constraints [23]. A key concern is that using a threshold that is too low (i.e., GDP per capita) may result in health systems choosing not to adopt an intervention that would generate net health benefits because the threshold does not take into account health opportunity costs [56]. Current efforts to develop country-specific estimates that account for opportunity costs, as well as updated data on population and economic growth, are underway and aim to provide better options for informing decision-making and resource allocation for health interventions $[24,25$, 56].

\section{Limitations}

The heterogeneity around reporting of costs and costing components made comparisons across 
studies challenging. For instance, some costing analyses accounted for all operational and personnel-related costs, and thus reported higher total costs, while other analyses report only direct costs related to diagnostics testing [11, 38]. There were no standardized screening algorithms, even across similar high-risk groups, and employed different standards of care (i.e., PCF or symptom screen alone or with CXR) which limits comparability of studies and generalizability to other settings. Not all studies describe cost components in the same manner [11] and comparisons across studies is further impeded by a range of primary outcomes from cost per case diagnosed, \$/ DALYs averted, \$/QALY, or \$/YLS. Thus, even among the studies that do calculate an ICER, direct comparison is not necessarily appropriate. No included studies assessed the impacts of screening on earlier case detection and proper TB treatment, but this is an area that merits additional evidence to better understand the impact on cost and cost-effectiveness of preventing additional disease transmission.

Our study was restricted to the published literature and thus is likely impacted by publication bias towards those interventions that were shown to be cost-effective; programmes that were not deemed cost-effective may not have been published. A recent Task Force Report from the Professional Society for Health Economics and Outcomes Research (ISPOR) suggests benchmarking approaches, such as reviewing trial protocols, to better explore the potential for publication bias but also notes the need to develop new approaches to assess publication bias [57]. Further, more recent economic evaluations of screening interventions, particularly for novel diagnostic tests such as Xpert ${ }^{\circledR}$, may have been more likely to demonstrate cost effectiveness than earlier studies due to consistently decreasing test costs.

Our review highlights key gaps in the existing economic evidence, namely the need for more studies on the costs and cost-effectiveness of systematic TB screening programmes from Latin America and Asia, since the majority of included studies took place in SSA. Access to various screening and diagnostic tools was not consistent across study settings. Increased efforts should be made to ensure availability of newer diagnostic technologies to TB programmes globally. In addition, standardization of systematic screening interventions, along with fixed and variable costs included in economic analyses of programmes, is needed for better evidence generation and comparability across studies.

\section{Conclusions}

The COVID-19 pandemic has dramatically impacted TB services globally. Modelling has shown that COVID-19 related restrictions and interruptions to $\mathrm{TB}$ programmes may result in an increase in TB incidence up to 6.3 million, and mortality to 1.5 million, by 2025 [58, 59]. Our review is the first to summarize the economic evidence for systematic screening for TB disease among high-risk groups. Our review highlights that to reach the "missing millions", and address the setbacks to TB services due to the COVID-19 pandemic, TB programmes should focus on simple, cheaper initial screening tools (i.e., symptom screen and CXR) followed by molecular diagnostic tools (i.e., Xpert ${ }^{\circledR}$ ) among the highest risk groups in the local setting (i.e., PLHIV, urban slums). Programmatic costs greatly impact cost-effectiveness thus future research should provide both fixed and variable costs of screening interventions to improve comparability. COVID-19 has dramatically increased the number of digital applications for contact screening, as well as other video or telehealth options for service delivery. Expanded use of such digital technologies can be leveraged for TB screening to improve identification and treatment options for patients globally.

\begin{abstract}
Abbreviations
W4SS: WHO-recommended four symptom screen; CRP: C-reactive protein; CXR: Chest X-ray; DALY: Disability-adjusted life year; DM: Diabetes mellitus; DOT: Directly-observed therapy; GDP: Gross domestic product; GNI: Gross national income; ICER: Incremental cost-effectiveness ratio; PCF: Passive case finding; PLHIV: People living with HIV; POC: Point of care; QALY: Qualityadjusted life year; SSA: Sub-Saharan Africa; SSM: Sputum smear microscopy; TB: Tuberculosis; WHO: World Health Organization; UR: Uncertainty range; USD: United States dollar; WTP:Willingness-to-pay; Xpert ${ }^{\circledR}$ : GeneXpert ${ }^{\circledR}$; YLG: Year of life gained; YLS: Year of life saved.
\end{abstract}

\section{Supplementary Information}

The online version contains supplementary material available at https://doi. org/10.1186/s12879-021-06633-3.

Additional file 1. Annex S1. Key PICO questions and full search terms used in systematic review.

\section{Acknowledgements}

We would like to thank Dr. Karen Steingart for her time in reviewing the manuscript and providing feedback.

\section{Authors' contributions}

AZ and $\mathrm{HA}$ wrote the study protocol. All authors (HA, BE and AZ) contributed to data extraction, analysis and interpretation. HA wrote the first version of the manuscript. All authors ( $\mathrm{HA}, \mathrm{BE}$, and $\mathrm{AZ}$ ) reviewed the manuscript and accepted the final version of the paper. All authors read and approved the final manuscript.

\section{Funding}

This study was supported by the Canadian Institutes of Health Research (\#168859) and the World Health Organization. This funding agencies had no role in study design, interpretation or writing of this report. The corresponding author had full access to all of the data and had final responsibility for the decision to submit for publication.

Availability of data and materials

Data sharing is not applicable to this article as no datasets were generated or analyzed during the current study. 


\section{Declarations}

Ethics approval and consent to participate

Not applicable.

\section{Consent for publication}

Not applicable.

\section{Competing interests}

We declare no competing interests.

\section{Author details}

${ }^{1}$ School of Epidemiology and Public Health, University of Ottawa, 600 Peter Morand Cresent, Ottawa, Canada. ${ }^{2}$ Children's Hospital of Eastern Ontario, Ottawa, Canada. ${ }^{3}$ Global TB Programme, World Health Organization, Geneva, Switzerland.

\section{Received: 17 February 2021 Accepted: 23 August 2021}

Published online: 08 September 2021

\section{References}

1. World Health Organization. Global tuberculosis report 2020. Geneva: WHO; 2020

2. Golub JE, Mohan $\mathrm{Cl}$, Comstock GW, et al. Active case finding of tuberculosis: historical perspective and future prospects. Int J Tuberc Lung Dis. 2005:9(11):1183-203.

3. WHO consolidated guidelines on tuberculosis: Module 2: screening-systematic screening for tuberculosis disease. Geneva2021.

4. Fox GJ, Johnston JC, Nguyen TA, et al. Active case-finding in contacts of people with TB. Int J Tuberc Lung Dis. 2021;25(2):95-105. https://doi.org/ 10.5588/ijtld.20.0658.

5. Kranzer K, Houben RM, Glynn JR, et al. Yield of HIV-associated tuberculosis during intensified case finding in resource-limited settings: a systematic review and meta-analysis. Lancet Infect Dis. 2010;10(2):93-102. https:// doi.org/10.1016/S1473-3099(09)70326-3.

6. Kuupiel D, Bawontuo V, Mashamba-Thompson TP. Mapping evidence on tuberculosis active case finding policies, strategies, and interventions for tuberculosis key populations: a systematic scoping review protocol. Syst Rev. 2019;8(1):162. https://doi.org/10.1186/s13643-019-1098-1.

7. Singh M, Sagili KD, Tripathy JP, et al. Are treatment outcomes of patients with tuberculosis detected by active case finding different from those detected by passive case finding? J Glob Infect Dis. 2020;12(1):28-33. https://doi.org/10.4103/jgid.jgid_66_19.

8. Shriraam V, Srihari R, Gayathri T, et al. Active case finding for Tuberculosis among migrant brick kiln workers in South India. Indian J Tuberc. 2020;67(1):38-42. https://doi.org/10.1016/j.ijtb.2019.09.003.

9. Jeon CY, Murray MB. Diabetes mellitus increases the risk of active tuberculosis: a systematic review of 13 observational studies. PLoS Med. 2008;5(7): e152. https://doi.org/10.1371/journal.pmed.0050152.

10. Sawert $H$, Kongsin S, Payanandana $V$, et al. Costs and benefits of improving tuberculosis control: the case of Thailand. Soc Sci Med. 1997;44(12):1805-16. https://doi.org/10.1016/s0277-9536(96)00289-4

11. Jit M, Stagg HR, Aldridge RW, et al. Dedicated outreach service for hard to reach patients with tuberculosis in London: observational study and economic evaluation. BMJ. 2011;343: d5376. https://doi.org/10.1136/bmj. d5376.

12. Silva EN, Pereira A, de Araujo WN, et al. A systematic review of economic evaluations of interventions to tackle tuberculosis in homeless people. Rev Panam Salud Publica. 2018;42: e40. https://doi.org/10.26633/RPSP. 2018.40.

13. Tavoschi L, Vroling H, Madeddu G, et al. Active case finding for communicable diseases in prison settings: increasing testing coverage and uptake among the prison population in the European union/European economic area. Epidemiol Rev. 2018;40(1):105-20. https://doi.org/10. 1093/epirev/mxy001.

14. Fox GJ, Barry SE, Britton WJ, et al. Contact investigation for tuberculosis: a systematic review and meta-analysis. Eur Respir J. 2013;41(1):140-56. https://doi.org/10.1183/09031936.00070812.
15. Zenner D, Southern J, van Hest R, et al. Active case finding for tuberculosis among high-risk groups in low-incidence countries. Int J Tuberc Lung Dis. 2013;17(5):573-82. https://doi.org/10.5588/ijtld.12.0920.

16. Dasgupta K, Menzies D. Cost-effectiveness of tuberculosis control strategies among immigrants and refugees. Eur Respir J. 2005;25(6):1107-16. https://doi.org/10.1183/09031936.05.00074004.

17. de Siqueira-Filha NT, Legood R, Cavalcanti A, et al. Cost of tuberculosis diagnosis and treatment in patients with HIV: a systematic literature review. Value Health. 2018;21(4):482-90. https://doi.org/10.1016/j.jval. 2017.09.003.

18. World Health Organization. Social determinants of health: Key concepts 2013. https://www.who.int/news-room/q-a-detail/social-determinantsof-health-key-concepts accessed November 232020.

19. Liberati A, Altman DG, Tetzlaff J, et al. The PRISMA statement for reporting systematic reviews and meta-analyses of studies that evaluate health care interventions: explanation and elaboration. Ann Intern Med. 2009;151(4):W65-94. https://doi.org/10.7326/0003-4819-151-4-20090 8180-00136.

20. Moher D, Shamseer $L$, Clarke $M$, et al. Preferred reporting items for systematic review and meta-analysis protocols (PRISMA-P) 2015 statement. Syst Rev. 2015;4:1. https://doi.org/10.1186/2046-4053-4-1.

21. Husereau D, Drummond M, Petrou S, et al. Consolidated health economic evaluation reporting standards (CHEERS) statement. BMJ. 2013;346: f1049. https://doi.org/10.1136/bmj.f1049.

22. Husereau D, Drummond M, Petrou S, et al. Consolidated health economic evaluation reporting standards (CHEERS)-explanation and elaboration: a report of the ISPOR health economic evaluation publication guidelines good reporting practices task force. Value Health. 2013;16(2):231-50. https://doi.org/10.1016/j.jval.2013.02.002.

23. Leech AA, Kim DD, Cohen JT, et al. Use and misuse of cost-effectiveness analysis thresholds in low- and middle-income countries: trends in costper-DALY studies. Value Health. 2018;21(7):759-61. https://doi.org/10. 1016/j.jval.2017.12.016

24. Marseille E, Larson B, Kazi DS, et al. Thresholds for the cost-effectiveness of interventions: alternative approaches. Bull World Health Organ. 2015;93(2):118-24. https://doi.org/10.2471/BLT.14.138206.

25. Bertram MY, Lauer JA, De Joncheere K, et al. Cost-effectiveness thresholds: pros and cons. Bull World Health Organ. 2016;94(12):925-30. https:// doi.org/10.2471/BLT.15.164418.

26. Organization $\mathrm{WH}$. New cost-effectiveness updates from $\mathrm{WHO}-\mathrm{CHOICE}$ Geneva: World Health Organization; https://www.who.int/news-room/ feature-stories/detail/new-cost-effectiveness-updates-from-who-choice accessed August 13, 2021.

27. Turner HC, Lauer JA, Tran BX, et al. Adjusting for inflation and currency changes within health economic studies. Value Health. 2019;22(9):102632. https://doi.org/10.1016/j.jval.2019.03.021.

28. World Data Inflation Rate Calculator Oldenburg, Germany. https://www. worlddata.info/ accessed June 292020.

29. Abimbola TO, Marston BJ, Date AA, et al. Cost-effectiveness of tuberculosis diagnostic strategies to reduce early mortality among persons with advanced HIV infection initiating antiretroviral therapy. J Acquir Immune Defic Syndr. 2012;60(1):e1-7. https://doi.org/10.1097/QAl.0b013e3182 $46538 f$.

30. Andrews JR, Lawn SD, Rusu C, et al. The cost-effectiveness of routine tuberculosis screening with Xpert MTB/RIF prior to initiation of antiretroviral therapy: a model-based analysis. AIDS. 2012;26(8):987-95. https:// doi.org/10.1097/QAD.0b013e3283522d47.

31. Bassett IV, Wang B, Chetty S, et al. Intensive tuberculosis screening for HIV-infected patients starting antiretroviral therapy in Durban. South Africa Clin Infect Dis. 2010;51(7):823-9. https://doi.org/10.1086/656282.

32. Bogdanova E, Mariandyshev O, Hinderaker SG, et al. Mass screening for active case finding of pulmonary tuberculosis in the Russian Federation: how to save costs. Int J Tuberc Lung Dis. 2019;23(7):830-7. https://doi. org/10.5588/ijtld.18.0449.

33. James $R$, Khim $K$, Boudarene $L$, et al. Tuberculosis active case finding in Cambodia: a pragmatic, cost-effectiveness comparison of three implementation models. BMC Infect Dis. 2017;17(1):580. https://doi.org/10. 1186/s12879-017-2670-8.

34. Ji Y, Cao H, Liu Q, et al. Screening for pulmonary tuberculosis in high-risk groups of diabetic patients. Int J Infect Dis. 2020;93:84-9. https://doi.org/ 10.1016/j.jij.2020.01.019. 
35. Jo Y, Mirzoeva F, Chry M, et al. Standardized framework for evaluating costs of active case-finding programs: an analysis of two programs in Cambodia and Tajikistan. PLoS ONE. 2020;15(1): e0228216. https://doi. org/10.1371/journal.pone.0228216.

36. Karki B, Kittel G, Bolokon I Jr, et al. Active community-based case finding for tuberculosis with limited resources. Asia Pac J Public Health. 2017;29(1):17-27. https://doi.org/10.1177/1010539516683497.

37. Kranzer K, Lawn SD, Meyer-Rath G, et al. Feasibility, yield, and cost of active tuberculosis case finding linked to a mobile HIV service in Cape Town, South Africa: a cross-sectional study. PLoS Med. 2012;9(8): e1001281. https://doi.org/10.1371/journal.pmed.1001281.

38. Machekera SM, Wilkinson E, Hinderaker SG, et al. A comparison of the yield and relative cost of active tuberculosis case-finding algorithms in Zimbabwe. Public Health Action. 2019;9(2):63-8. https://doi.org/10.5588/ pha.18.0098.

39. Maheswaran H, Barton P. Intensive case finding and isoniazid preventative therapy in HIV infected individuals in Africa: economic model and value of information analysis. PLoS ONE. 2012;7(1): e30457. https://doi. org/10.1371/journal.pone.0030457.

40. Murray M, Cattamanchi A, Denkinger C, et al. Cost-effectiveness of triage testing for facility-based systematic screening of tuberculosis among Ugandan adults. BMJ Glob Health. 2016;1(2): e000064. https://doi.org/10. 1136/bmjgh-2016-000064.

41. Orlando S, Triulzi I, Ciccacci F, et al. Delayed diagnosis and treatment of tuberculosis in HIV+ patients in Mozambique: a cost-effectiveness analysis of screening protocols based on four symptom screening, smear microscopy, urine LAM test and Xpert MTB/RIF. PLoS ONE. 2018;13(7): e0200523. https://doi.org/10.1371/journal.pone.0200523.

42. Reddy KP, Gupta-Wright A, Fielding KL, et al. Cost-effectiveness of urinebased tuberculosis screening in hospitalised patients with HIV in Africa: a microsimulation modelling study. Lancet Glob Health. 2019;7(2):e200-8. https://doi.org/10.1016/S2214-109X(18)30436-4.

43. Sekandi JN, Dobbin K, Oloya J, et al. Cost-effectiveness analysis of community active case finding and household contact investigation for tuberculosis case detection in urban Africa. PLOS ONE. 2015;10(2): e0117009. https://doi.org/10.1371/journal.pone.0117009.

44. Shah NS, Anh MH, Thuy TT, et al. Population-based chest X-ray screening for pulmonary tuberculosis in people living with HIV/AIDS, An Giang, Vietnam. Int J Tuberc Lung Dis. 2008;12(4):404-10.

45. Shah S, Demissie M, Lambert L, et al. Intensified tuberculosis case finding among HIV-Infected persons from a voluntary counseling and testing center in Addis Ababa. Ethiopia J Acquir Immune Defic Syndr. 2009;50(5):537-45. https://doi.org/10.1097/QAl.0b013e318196761c.

46. Shah L, Rojas M, Mori O, et al. Cost-effectiveness of active case-finding of household contacts of pulmonary tuberculosis patients in a low HIV, tuberculosis-endemic urban area of Lima. Peru Epidemiol Infect. 2017;145(6):1107-17. https://doi.org/10.1017/S0950268816003186.

47. Smit GS, Apers L, Arrazola de Onate W, et al. Cost-effectiveness of screening for active cases of tuberculosis in Flanders, Belgium. Bull World Health Organ. 2017;95(1):27-35. https://doi.org/10.2471/BLT.16.169383.
48. Winetsky DE, Negoescu DM, DeMarchis EH, et al. Screening and rapid molecular diagnosis of tuberculosis in prisons in Russia and Eastern Europe: a cost-effectiveness analysis. PLoS Med. 2012;9(11): e1001348. https://doi.org/10.1371/journal.pmed.1001348.

49. Yoon C, Semitala FC, Asege $L$, et al. Yield and efficiency of novel intensified tuberculosis case-finding algorithms for people living with HIV. Am J Respir Crit Care Med. 2019;199(5):643-50. https://doi.org/10.1164/rccm. 201803-04900C.

50. Zhang C, Ruan Y, Cheng J, et al. Comparing yield and relative costs of WHO TB screening algorithms in selected risk groups among people aged 65 years and over in China, 2013. PLoS ONE. 2017;12(6): e0176581. https://doi.org/10.1371/journal.pone.0176581.

51. Zishiri V, Charalambous S, Shah MR, et al. Implementing a large-scale systematic tuberculosis screening program in correctional facilities in South Africa. Open Forum Infect Dis. 2015;2(1):ofu121. https://doi.org/10. 1093/ofid/ofu121.

52. Zwerling AA, Sahu M, Ngwira LG, et al. Screening for tuberculosis among adults newly diagnosed with HIV in sub-saharan Africa: a cost-effectiveness analysis. J Acquir Immune Defic Syndr. 2015;70(1):83-90. https://doi. org/10.1097/QAl.0000000000000712.

53. Adelman MW, McFarland DA, Tsegaye M, et al. Cost-effectiveness of WHO-recommended algorithms for TB case finding at Ethiopian HIV clinics. Open Forum Infect Dis. 2018;5(1):ofx269. https://doi.org/10.1093/ofid/ ofx269.

54. Sohn H, Vyas A, Puri L, et al. Costs and operation management of community outreach program for tuberculosis in tribal populations in India. Public Health Action. 2019;9(2):58-62. https://doi.org/10.5588/pha.18. 0091.

55. Systematic Screening for Active Tuberculosis: Principles and Recommendations. Geneva2013.

56. Ochalek J, Claxton K, Lomas J, et al. Valuing health outcomes: developing better defaults based on health opportunity costs. Expert Rev Pharmacoecon Outcomes Res. 2020. https://doi.org/10.1080/14737167.2020.18123 87.

57. Mandrik OL, Severens JLH, Bardach A, et al. Critical appraisal of systematic reviews with costs and cost-effectiveness outcomes: an ISPOR good practices task force report. Value Health. 2021;24(4):463-72. https://doi. org/10.1016/j.jval.2021.01.002

58. Cilloni L, Fu H, Vesga JF, et al. The potential impact of the COVID-19 pandemic on the tuberculosis epidemic a modelling analysis. EClinicalMedicine. 2020;28: 100603. https://doi.org/10.1016/j.eclinm.2020.100603.

59. Sahu S, Ditiu L, Sachdeva KS, et al. Recovering from the impact of the Covid-19 pandemic and accelerating to achieving the United Nations general assembly tuberculosis targets. Int J Infect Dis. 2021. https://doi. org/10.1016/j.jid.2021.02.078[publishedOnlineFirst:2021/03/16].

\section{Publisher's Note}

Springer Nature remains neutral with regard to jurisdictional claims in published maps and institutional affiliations.
Ready to submit your research? Choose BMC and benefit from:

- fast, convenient online submission

- thorough peer review by experienced researchers in your field

- rapid publication on acceptance

- support for research data, including large and complex data types

- gold Open Access which fosters wider collaboration and increased citations

- maximum visibility for your research: over 100M website views per year

At BMC, research is always in progress.

Learn more biomedcentral.com/submissions 